\title{
New developments in sustained release drug delivery for the treatment of intraocular disease
}

\author{
Gisela Velez, Scott M Whitcup
}

Over the past 20 years, drug development has led to numerous medications with the potential to treat intraocular disease. These medications usually require systemic or frequent topical administration, which can limit the types of medications that can be used and the diseases that can be treated effectively.

\section{Current methods of ocular drug delivery: limitations}

Topical administration of medications can successfully treat a number of ocular diseases such as glaucoma, inflammation of the anterior segment or ocular surface, and external diseases of the eye. Nevertheless, topical delivery often fails to provide therapeutic levels in the vitreous cavity or posterior segment, and therefore is inadequate in the treatment of vitreoretinal diseases.

Drugs can often be delivered to the posterior segment by injection via the pars plana, as is the case, for example, with ganciclovir, foscarnet, and cidofovir. However, depending on the rate of clearance from the vitreous of a particular medication, large boluses and frequent administrations may be required to ensure therapeutic levels over an extended period of time. Frequent injections in clinical practice may not be practical for chronic diseases that can sometimes require multiple weekly administrations over months or years. In addition, multiple intraocular injections can lead to an increased likelihood of complications, such as vitreous haemorrhage, retinal detachment, and endophthalmitis.

The blood-ocular barrier is the main obstacle in the treatment of intraocular disease with systemic medications. Poor penetration of many drugs into the eye not only limits the number of medications available for the treatment of ocular diseases, but also limits the extent to which those available can be used without incurring serious systemic side effects. Medications, such as prednisone, cytotoxic agents for the treatment of intraocular inflammation, and antivirals for the treatment of cytomegalovirus (CMV) retinitis often cause severe side effects at the dosages needed to achieve their desired therapeutic effect. This is particularly problematic when the drugs can cause severe morbidity and mortality and the disease is limited to the eye.

Manipulation of the blood-ocular barrier can be used to increase drug permeability. Two main sites of the blood-ocular barrier exist: the blood-aqueous barrier and the blood-retinal barrier. The blood-aqueous barrier is formed by the non-pigmented layer of the ciliary epithelium and the endothelium of the iris vessels, and controls mostly inward movement from the blood to the eye. The blood-retinal barrier is formed by the retinal pigment epithelium and the endothelium of the retinal vessels and affects mostly outward movement from the retina to the blood. ${ }^{1-4}$ Passive diffusion, particularly in the bloodretinal barrier, is extremely restricted.

Intracarotid or ophthalmic artery perfusion of hypertonic substances, also known as barrier "disruption", increases permeability of the blood-retinal barrier in two ways: by causing a reversible widening of endothelial tight junctions through osmotic shrinkage of the cells, and by affecting the basal tight junctions of the retinal pigment epithelium. In the ciliary epithelium, osmotic disruption causes separation of the non-pigmented epithelium from the pigmented epithelium, disruption of the tight junctions between non-pigmented epithelial cells, and widening of endothelial fenestrations in ciliary capillaries. Though some of the ultrastructural effects can be permanent, the physiological effects observed are always reversible. ${ }^{56}$

Permeability of the blood-ocular barrier also varies depending on drug characteristics. It is higher for substances that are lipophilic or have greater oil/water ratios. Non-lipophilic antibiotics, such as penicillins, cephalosporins, and aminoglycosides, have been shown to achieve intavitreal levels no higher than $10 \%$ of serum levels after venous infusion, even in the presence of inflammation. ${ }^{7}$ Other, more lipophilic, antibiotics such as chloramphenicol and doxycycline, have been shown to readily traverse the blood-ocular barrier. $^{7}$ Lipophilic analogues or prodrugs of compounds with poor penetrance, therefore, can be devised to improve blood-retinal barrier permeability.

Blood barrier disruption and the synthesis of lipophilic prodrugs have disadvantages, however. Intraocular levels with blood-ocular barrier disruption can be difficult to predict, and may vary widely among patients. In addition, systemic administration of hypertonic agents for bloodocular barrier disruption is often poorly tolerated by patients and can lead to systemic side effects. Although the design of lipophilic prodrugs is a viable alternative, the time spent developing these analogues can delay for years the diversity and number of medications available for the treatment of ocular disease.

\section{Local intraocular drug delivery: advantages}

Advances in materials sciences and surgical technique have allowed the development of a drug delivery system that can overcome the limitations of topical and systemic routes of administration. Implants can now be produced with a core of drug surrounded by layers of permeable and impermeable polymers. These implants can then be placed in the vitreous or subconjunctivally to deliver drug to the eye. They can also be attached to intraocular lenses placed during cataract surgery as another method of delivery.

Local delivery of drugs to the eye via intravitreal implants offers several advantages over systemic therapy. Firstly, it bypasses the blood-ocular barriers, allowing 
higher intraocular drug levels than could be achieved by systemic or topical administration. Secondly, it avoids many of the side effects associated with systemic therapy. This is particularly of benefit in the case of medications that may be too toxic for systemic administration but would be well tolerated by the eye. A good example is trifluoridine (Viroptic, Wellcome). Originally developed for the systemic treatment of herpetic infections, it was found to be mutagenic, carcinogenic, and teratogenic with systemic administration. Dosages as low as $1.5 \mathrm{mg} / \mathrm{kg}$ a day resulted in an increased risk of carcinomas and sarcomas in animals. ${ }^{8}$ However, topical administration for the treatment of herpes simplex keratoconjunctivitis and epithelial keratitis is effective and well tolerated by the eye, without the systemic exposure and increased risk of neoplasia.

Intravitreal implants also provide relatively constant drug levels in the eye. Their release rates can be carefully controlled to avoid subtherapeutic or toxic levels. This is particularly valuable when local or systemic therapy mandates intravenous administration or frequent dosing; or when patient compliance is of concern. Finally, much less drug is needed for this local drug delivery, which is especially important for new therapeutic agents that may be in short supply or extremely expensive.

\section{Local intraocular drug delivery: disadvantages}

There are, however, some disadvantages to intravitreal implant therapy. Local drug delivery to a single eye does not treat the contralateral eye. Furthermore, for diseases not limited to the eye, local drug delivery fails to treat extraocular disease. This is particularly the case with infectious diseases such as CMV, or ocular inflammatory diseases with systemic involvement, such as Behçet's disease, Vogt-Kayanagi-Harada disease, and sarcoidosis. Higher intravitreal concentrations of drugs may offer a greater therapeutic effect, but may also be associated with increased ocular toxicity. Drugs that may be safe to the eye when used for a short time may prove to be toxic when allowed to maintain long standing intraocular levels. Finally, surgical placement of intravitreal implants can cause adverse effects including vitreous haemorrhage, retinal detachment, and endophthalmitis.

This review documents the technological development of sustained release intravitreal implants, and reviews their use in ocular disease. The ganciclovir implant has been approved by the Food and Drug Administration and used successfully for several years. Several intraocular implants are currently under investigation in clinical trials, and others are undergoing preclinical testing.

\section{Sustained release intravitreal ganciclovir implant} Sustained release local drug delivery has had a profound impact on the treatment of cytomegalovirus retinitis. Before the use of highly active antiretroviral therapy (HAART), treatment of cytomegalovirus required lifelong systemic medications. Unfortunately, systemic administration of ganciclovir, foscarnet, or cidofovir is associated with significant side effects. Ganciclovir and foscarnet can cause myelosuppression and renal toxicity respectively. ${ }^{9-12} \mathrm{Be}-$ cause of concern over the toxicity of systemic administration of anti-CMV therapy, ophthalmologists began injecting drugs directly into the vitreous. Henry and coworkers first reported the treatment of CMV retinitis with intravitreal injections of ganciclovir. ${ }^{13}$ However, the relatively short half life of ganciclovir in the eye required one to two weekly injections to maintain therapeutic drug levels. The need for multiple injections is not only inconvenient for patients, but also increases the risk of ocular complications. ${ }^{14-16}$

The development of a sustained release ganciclovir implant has allowed local drug delivery without the need

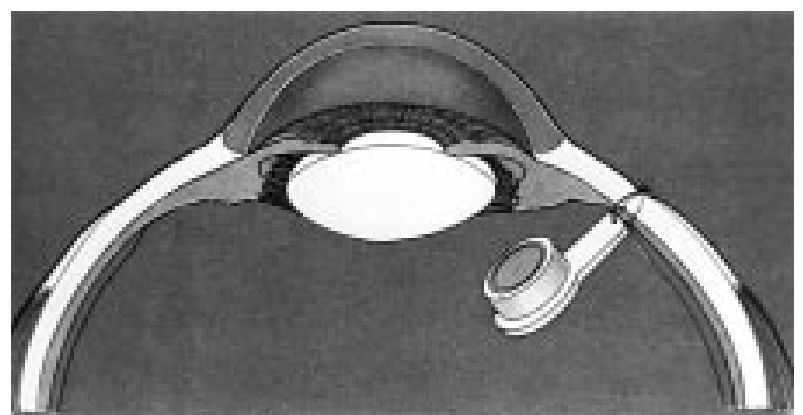

Figure 1 Schematic representation of intravitreal implant fixed to the sclera at the pars plana (reprinted from Sanborn et al, ${ }^{20}$ with permission from Archives of Ophthalmology).

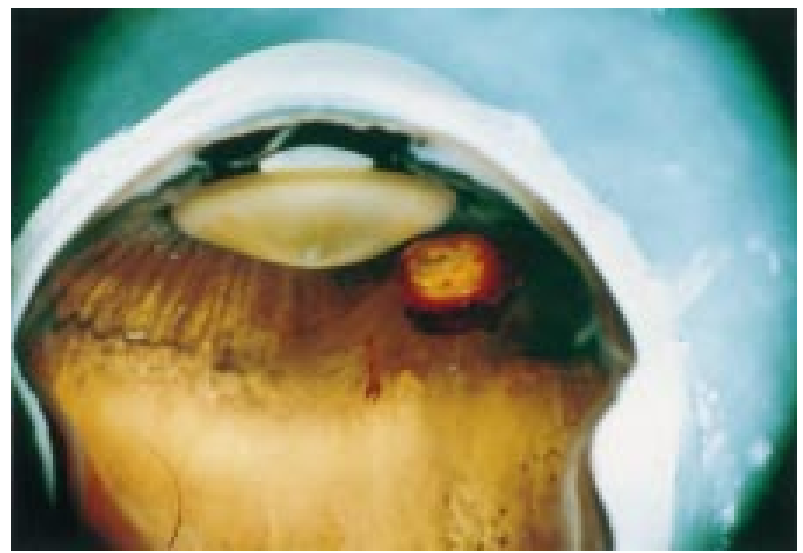

Figure 2 Gross section of a human eye showing an intraocular ganciclovir implant at the pars plana (courtesy of Dr Chi-Chao Chan, National Eye Institute, $N I H$ ).

for multiple injections. The ganciclovir implant is produced by partially coating a compressed pellet of ganciclovir with ethylene vinyl acetate, a compound that is impermeable to the drug, and a surrounding coat of polyvinyl alcohol. ${ }^{17}$ This implant releases drug at a rate of $1 \mu \mathrm{g} / \mathrm{h}$ and leads to intravitreal drug levels that are much higher than those achieved with maintenance intravenous therapy. ${ }^{18} 19$ Current implants deliver drug for approximately 8 months. ${ }^{20}{ }^{21}$ The implant is surgically placed through the pars plana, and has been well tolerated in the eye (Figs 1 and 2).

The ganciclovir implant has been shown to be effective for treating CMV retinitis in randomised clinical trials. In a National Eye Institute clinical trial, patients with active peripheral CMV retinitis were randomly assigned to immediate treatment with a ganciclovir implant or to deferred treatment. ${ }^{19}$ The median time to progression of retinitis was 15 days for patients in the deferred treatment group and 226 for patients receiving the ganciclovir implant $(\mathrm{p}<0.0001)$. Postoperative complications included mild vitreous haemorrhage, seven late retinal detachments, and one retinal tear without retinal detachment. In a second randomised clinical trial, the median time to progression of retinitis was 71 days in patients treated with intravenous ganciclovir and 221 days in patients receiving ganciclovir implants $(\mathrm{p}<0.001) .^{22}$

Although the ganciclovir implant is more effective for the treatment of CMV retinitis, the implant does not treat systemic CMV infection or prevent CMV retinitis in the contralateral eye. In a study of 377 patients with AIDS and unilateral CMV retinitis, patients were randomly assigned to receive a ganciclovir implant plus oral ganciclovir, a ganciclovir implant plus oral placebo, or intravenous ganciclovir alone. ${ }^{23}$ The incidence of new CMV disease at 6 months was $44.3 \%$ in the group assigned to the 
ganciclovir implant plus placebo compared with $24.3 \%$ in the group assigned to the ganciclovir implant plus oral ganciclovir $(p=0.002)$ and $19.6 \%$ in the group assigned to intravenous ganciclovir $(\mathrm{p}<0.001)$.

Sustained release intravitreal cyclosporin implant Cyclosporin is an effective secondary agent in the treatment of uveitis. Usually reserved for patients with advanced bilateral disease despite high doses of prednisone, its main effect is on the recruitment and activation of $T$ cells. It is believed to act by interfering with interleukin 2 (IL-2) in the activation of T cell genes. ${ }^{24}{ }^{25}$ Although CD4 lymphocytes are the main target, CD8 cells are also suppressed. Systemic administration is usually through the oral route as a suspension. The gastrointestinal absorption is variable, however, with a range of $4-60 \%{ }^{26}$ Some of the most common side effects at a dose of 10 $\mathrm{mg} / \mathrm{kg}$ include paraesthesias and hyperaesthesia (40\%), hypertension $(24 \%)$, epigastric burning (20\%), hypertrichorism and gingivitis $(20 \%) .{ }^{27}$ Hypertension, renal toxicity, and an increase in the predisposition to malignancy such as lymphoma are perhaps the most threatening. Many patients require long term management, increasing the risk of complications and making careful monitoring of their renal function, blood pressure, and surveillance for malignancy an important part of their management. In addition, baseline abnormalities in renal function tests often preclude the use of cyclosporin in certain patients.

Studies in rabbits have demonstrated that the intravitreal administration of $100 \mu \mathrm{g}$ of cyclosporin is non-toxic to retinal structures. ${ }^{28}$ Intravitreal injections of cyclosporin have been used in the rat model to treat EAU (experimental autoimmune uveitis) without significant blood levels. ${ }^{29}$ However, the intraocular half life of cyclosporin would require multiple weekly injections, making such a delivery impractical. ${ }^{30}$

An implant has been designed for the long term intravitreal release of cyclosporin A. It bypasses the systemic circulation, avoiding the side effects, while administering therapeutic doses of the medication to the eye over an extended period of time. The implant consists of a drug pellet coated with silicone attached to a polyvinyl alcohol anchor strut. This design has been used to create implants that can release the drug at several rates, depending on the material used to coat the drug pellet. Experimental studies have shown that devices releasing cyclosporin at a rate of $1.3 \mu \mathrm{g} /$ day can achieve intravitreal levels over a 6 month period of $500 \mathrm{ng} / \mathrm{ml}$, or five times the therapeutic level needed to suppress $\mathrm{T}$ cell activation. ${ }^{30}{ }^{31}$ Systemic doses of at least $5 \mathrm{mg} / \mathrm{kg}$ are usually necessary to achieve intravitreal levels of $100 \mathrm{ng} / \mathrm{ml}^{32}$

Animal studies have demonstrated the efficacy of this delivery system in the control of intraocular inflammation in a rabbit model of uveitis. ${ }^{33} \mathrm{~A}$ phase I/II clinical trial is currently under way at the National Eye Institute and Duke University. Implants of 1 or $2 \mathrm{mg}$ with a release rate of 0.8 or $1.4 \mu \mathrm{g} / \mathrm{ml}$ respectively are being evaluated in patients with uveitis. Both implants have been shown empirically to release cyclosporin $\mathrm{A}$ at rates which achieve therapeutic intravitreal levels for up to 10 years (Fig 3).

\section{Sustained release intravitreal corticosteroid implants}

Corticosteroids play an important part in the current management of ocular inflammatory disease. Topical, periocular, or systemic administrations remain the mainstay of treatment. Corticosteroids also may have an important role in the management of ocular diseases that involve neovascularisation, wound healing, and fibrosis, such as proliferative vitreoretinopathy and subretinal neovascularisation.

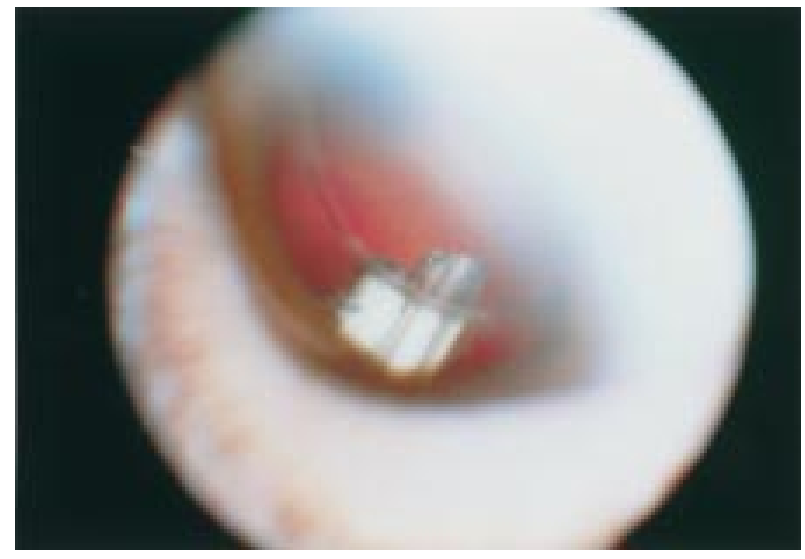

Figure 3 Slit lamp photograph showing a cyclosporin implant in the inferotemporal quadrant. The patient is pseudophakic.

Experiments on a rabbit model for posterior vitreoretinopathy have shown that an intraocular injection of dexamethasone can successfully inhibit fibrous proliferation in the vitreous cavity. ${ }^{34}$ This inhibitory effect on fibroblasts has also been confirmed through in vitro studies on fibroblast proliferation, ${ }^{35}{ }^{36}$ and has been observed in endothelial cells and neovascularisation. ${ }^{37}$ The dosages noted to achieve such effects are $200 \mu \mathrm{g} / \mathrm{ml}$ for dexamethasone sodium phosphate and $150 \mu \mathrm{g} / \mathrm{ml}$ for triamcinolone acetonide. These dosages, however, are much higher than could be achieved by systemic or periocular administration. ${ }^{38} \mathrm{~A}$ study by Weijtens et al showed that the peribulbar injection of $5 \mathrm{mg}$ of dexamethasone phosphate resulted in a peak intravitreal concentration of $0.013 \mu \mathrm{g} / \mathrm{ml} .^{39}$ Similarly, a study by the same group showed the median intravitreal concentration of dexamethasone phosphate after oral intake of $7.5 \mathrm{mg}$ to be $0.0052 \mu \mathrm{g} / \mathrm{ml}^{40}$

Intraocular delivery of corticosteroids could play in important part in the treatment of ocular diseases which involve inflammation, fibrous proliferation, and neovascularisation by sustaining intravitreal steroid levels which could otherwise not be achieved. A dexamethasone implant has been shown, for example, to effectively inhibit the development of experimental uveitis in a rabbit model. ${ }^{41}$ In this experiment, the implant was able to deliver therapeutic doses for 99 days, after which $30 \%$ of the drug still remained. A fluocinolone intravitreal sustained release device has also been shown to inhibit experimental choroidal neovascularisation. ${ }^{42}$ The possible side effects of this therapy include glaucoma and cataract formation, and should not be ignored. Clinical trials at the National Eye Institute are currently being implemented to investigate the safety and potential effectiveness of these implants in the treatment of uveitis and age related macular degeneration.

Co-drug implants can also be created which combine the antiproliferative effects of steroids and compounds with similar properties such as fluorouracil. Recently, Yang and co-workers demonstrated that a sustained release triamcinolone and 5-fluorouracil co-drug was effective in the inhibition of proliferative vitreoretinopathy in a rabbit model. ${ }^{43} \mathrm{~A}$ similar implant consisting of fluocinolone and 5-fluorouracil has been tested in the same model with similar results. $^{44}$

\section{Other applications}

Sustained release technology allows the local delivery of a number of drugs for the treatment of ocular disease. Compounds that are potentially effective yet restricted by their systemic side effects can now be reconsidered as treatments. Angiostatic agents, for example, are currently under 


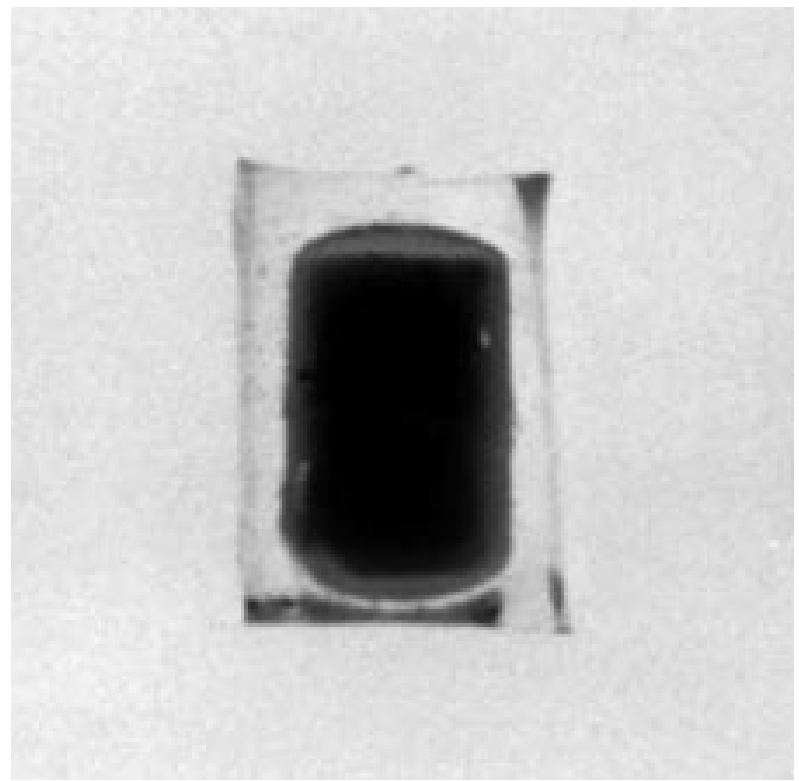

Figure 4 Experimental thalidomide implant, $10 \mathrm{mg}$ pellet, with a silicone shell.

investigation for the treatment of age related macular degeneration. Despite the potential benefits, systemic administration of these agents can have significant side effects. Thalidomide, for example, is known to be a powerful angiostatic agent. Its systemic side effects, however, include peripheral neuropathy, central nervous system depression, and embryotoxicity, and have limited the dosages administered to patients for the treatment of subretinal neovascularisation. Systemic inhibition of angiogenesis in older patients can also interfere with the development of collateral circulation, which has a role in the prevention of central nervous system as well as cardiac ischaemic events. Recently, we developed an implant that successfully releases therapeutic intraocular doses of thalidomide, and could be used for the treatment of subretinal neovascularisation (Fig 4). ${ }^{45}$

Implants have already been developed for other applications. An intravitreal implant, for example, has been developed for the sustained release of calcium channel blockers for the potential treatment of glaucoma. ${ }^{46}$ Recent data have suggested that calcium channel antagonists may improve optic nerve head circulation as well as improve visual field testing in patients with normal tension glaucoma. ${ }^{47-49} \mathrm{An}$ implant that can deliver calcium channel antagonists directly to the eye could avoid systemic haemodynamic effects such as hypotension.

An intravitreal implant of trimetrexate has also been developed for the treatment of intraocular lymphoma. ${ }^{50}$ Like its parent drug, methotrexate, trimetrexate inhibits the enzyme dihydrofolate reductase, thereby interfering with the production of reduced folate cofactors. Certain cellular reactions responsible for the production of nucleotides are dependent on these cofactors. ${ }^{51}$ Trimetrexate, therefore, interferes with DNA and RNA synthesis (S phase) of the cell, making it an effective agent against rapidly dividing lymphoma cells. Systemic administration, however, can result in haematological, hepatic, renal, and gastrointestinal toxicity requiring the concomitant administration of leucovorin. In addition, the systemic clearance of trimetrexate can be very rapid, with a terminal serum half life of less than 12 hours. ${ }^{52}$ Frequent systemic administrations at high doses would be required for the treatment of intraocular disease, and the development of an intraocular implant could clearly be of benefit in these patients.
Other novel designs such as the use of nanospheres or subconjunctival matrices will provide more flexibility in the application of this technology, allowing the mode of drug delivery to be customised to the particular properties of a compound and the disease being treated. Nanospheres and other vehicles made of biodegradable materials will allow sustained release drug formulations to be injected into the eye. Their short life spans make them ideal for use in diseases such as infectious endophthalmitis, retinopathy of prematurity, and post-traumatic vitreous proliferation. Lipophilic prodrugs can also be formulated as liposomal preparations, and can act as natural microsomes and prolong intravitreal concentrations. Such preparations could be injected intravitreally, and achieve therapeutic concentrations for short though significant periods of time. Recently, Cheng and colleagues published encouraging data on such a formulation of a lipid prodrug of foscarnet for the treatment of CMV retinitis. ${ }^{53}$ Subconjunctival matrices could be the delivery of choice for highly lipophilic agents which more easily penetrate the eye and do not require intraocular administration to achieve therapeutic levels.

Finally, gene therapy may be a unique technology allowing long term delivery of therapeutic agents. Viral vectors can be inserted into the eye for the transfection of genes to specific tissues. Genes coding for specific proteins can be incorporated into cells, leading to the long term expression of proteins or enzymes which are deficient or absent in a particular disease, or to the normalisation of gene expression in diseases where overproduction leads to pathology. Such genetic manipulation would allow effective treatment of ocular disease with minimal surgical intervention. However, this technology has a number of drawbacks in its current development. Disease mechanisms must be clearly established and the involved genes identified before this approach is applicable. Viral vectors can be used to target specific ocular tissues and have been shown to deliver genetic material effectively, ${ }^{54}$ but are limited by the immune response targeted against the virus, their transduction efficiency, the size of the DNA they can deliver, and the length of gene expression. ${ }^{55}$ Non-viral vectors may be safer and less costly, but are limited by their poor transfection rates and expression efficiency. Current research in this field, however, is making this technology a promising and plausible treatment modality for ocular disease.

\section{Conclusion}

Recent advances in implant technology make possible the design of implants that can release a wide range of medications for predictable periods of time. The technology opens new therapeutic options for intraocular disease by allowing a practical way to deliver both old and new drugs to the eye. Experimental and clinical trials under way will further define the benefits and limitations of this exciting new technology.

GISELA VELEZ SCOTT M WHITCUP

National Eye Institute, National Institutes of Health, Bethesda, MD, USA

Correspondence to: Gisela Velez, MD, MPH, National Eye Institute, NIH, 10 Center Drive, Bldg 10, Rm 10N112, Bethesda, MD 20892, USA

1 Peyman GA, Schulman, JA. Intravitreal drug therapy. Fpn $\mathcal{f}$ Ophthalmol 1989;33:392-404.

2 Cunha-Vaz JG. The blood-ocular barriers: past, present, and future. Doc Ophthalmol 1997;93:149-57.

3 Cunha-Vaz JG. The blood-ocular barriers. Surv Ophthalmol 1979;23:27996.

4 Foulds WS, Moseley H, Eadie A, et al. Vitreal, retinal, and pigment epithelial contributions to the posterior blood-ocular barrier. Trans Ophthalmol Soc UK 1980;100:341-2.

5 Rapoport SI. Osmotic opening of blood-brain and blood-ocular barriers. Exp Eye Res 1977;(Suppl):499-509. 
6 Laties AM, Rapoport SI. The blood-ocular barriers under osmotic stress: studies on the freeze-dried eye. Arch Ophthalmol 1976;94:1086-91.

7 Barza M. Factors affecting the intraocular penetration of antibiotics: the influence of route, inflammation, animal species and tissue pigmentation. Scand F Infect Dis 1978;(Suppl) 14:151-9.

8 Physician's desk reference. 52 nd ed. Montvale, NJ: Medical Economics Company, 1998:1795-6.

9 Spector SA, Weingeist T, Pollard RB, et al. A randomized, controlled study of intravenous ganciclover therapy for cytomegalovirus peripheral retinitis in patients with AIDS. AIDS Clinical Trials Group and Cytomegalovirs Cooperative Study Group. F Infect Dis 1993;168:557-63.

10 Hochster H, Dietrich D, Bozzette S, et al. Toxicity of combined ganciclovir and zidovudine for cytomegalovirus disease associated with AIDS. An AIDS Clinical Trials Group Study. Ann Intern Med 1990;113:111-7.

11 Palestine AG, Polis MA, De Smet MD, et al. A randomized, controlled trial of foscarnet in the treatment of cytomegalovirus retinitis in patients with AIDS. Ann Intern Med 1991;115:665-73.

12 Masur H, Whitcup SM, Cartwright C, et al. Advances in the management of AIDS-related cytomegalovirus retinitis. Ann Intern Med 1996;125:126-36.

13 Henry K, Cantrill HL, Fletcher C, et al. Use of intravitreal ganciclovir Henry K, Cantrill HL, Fletcher C, et al. Use of intravitreal ganciclovir
(dihydroxypropoxymethyl guanine) for cytomegalovirus retinitis in a (dihydroxypropoxymethyl guanine) for cytomegaloviru

14 Heinemann MH. Long-term intravitreal ganciclovir therapy for cytomegalovirus retinopathy. Arch Ophthalmol 1989;107:17

15 Cantrill H, Henry K, Melroe NH, et al. Treatment of cytomegalovirus retinitis with intravitreal ganciclovir: long-term results. Ophthalmology 1989;96:367-74

16 Cochereau-Massin I, Le Hoang P, Lautier-Frau M, et al. Efficacy and tolerance of intravitreal ganciclovir in cytomegalovirus retinitis in acquired immune deficiency syndrome. Ophthalmology 1991;98:1348-53.

17 Smith TJ, Pearson TA, Blanford DL, et al. Intravitreal sustained-release ganciclovir. Arch Ophthalmol 1992;110:255-8.

18 Kupperman BD, Quiceno JI, Flores-Aguilar M, et al. Intravitreal ganciclovir concentration after intravenous administration in AIDS patients with cytomegalovirus retinitis: implications for therapy. F Infect Dis 1993;168: 1506-9.

19 Martin DF, Parks DJ, Mellow SD, et al. Treatment of cytomegalovirus retinitis with an intraocular sustained-release ganciclovir implant. A randretinitis with an intraocular sustained-release ganciclovir implant. A
omized, controlled clinical trial. Arch Ophthalmol 1994;112:1531-9.

20 Sanborn GE, Anand R, Tori RE, et al. Sustained-release ganciclovir therapy for treatment of cytomegalovirus retinitis: use of an intravitreal device. Arch Ophthalmol 1992;110:188-95.

21 Anand R, Nightingale SD, Fish RH, et al. Control of cytomegalovirus retinitis using sustained release of intraocular ganciclovir. Arch Ophthalmol 1993; 111:223-7.

22 Musch DC, Martin DF, Gordon JF, et al. Treatment of cytomegalovirus retinitis with a sustained-release ganciclovir implant. $N$ Engl $\mathcal{F} \mathrm{Med}$ 1997;337:83-90.

23 Martin DF, Kuppermann DB, Wolitz RA, et al. Oral ganciclovir in cytomegalovirus retinitis treated with a ganciclovir implant. $N$ Engl f $\mathrm{Med}$ 1999;340:1063-70.

24 Granelli-Piperno A, Nolan P, Inaba K et al. The effect of immunosuppressive agents on the induction of nuclear factors that bind to sites on the interleukin 2 promotor. $\mathcal{F}$ Exp Med 1990;172:1869-72.

25 Larsson AL. Cyclosporine A and dexamethasone suppress T-cell responses by selectively acting at distinct sites of the triggering process. F Immunol 1980;124:2828-33.

26 Beveridge T, Gratwohl A, Michot F et al. Cyclosporine A: pharmacokinetics after a singel dose in arm and serum levels after multiple dosing in recipients of allogeneic bone-marrow grafts. Curr Ther Res 1981;30:5-18.

27 Nussenblatt R, Whitcup SM, Palestine A. Uveitis: fundamental and clinical practice. 2nd ed. St Louis: Mosby, 1996:116.

28 Grisolano J Jr, Peyman GA. Retinal toxicity of intravitreal cyclosporine. Ophthalmic Surg 1986;17:155-6.

29 Nussenblatt RB, Dinning WJ, Fujikawa LS, et al. Local cyclosporine therapy for experimental autoimmune uveitis in rats. Arch Ophthalmol 1985;103: 1559-62.

30 Pearson AP, Jaffe GF, Martin DF et al. Evaluation of a delivery system providing long term release of cyclosporine. Arch Ophthalmol 1996;114:311-7.
31 Laferty KJ. Cyclosporin-A: models for the mechanism of action. Transplant Proc 1983;15:2242-7.

32 BenEzra D, Maftzir G, deCourten C, et al. Ocular penetration of cyclosporin-A, III: the human eye. Br f Ophthalmol 1990;74:350-2.

33 Jaffe GJ, Yang CS, Wanx XC, et al. Intravitreal sustained-release cyclosporin A in the treatment of experimental uveitis. Ophthalmology 1998;105:46-56.

34 Tano Y, Sugita G, Abrams G, et al. Inhibition of intraocular proliferations with intravitreal corticosteroids. Am f Ophthalmol 1980;89:131-6.

35 Grossfeld H, Ragan C. Action of hydrocortisone on cells in tissue culture. Proc Soc Exp Biol Med 1954;86:63.

36 Blumenkranz MS, Claflin A, Hajek A. Selection of therapeutic agents for intraocular proliferative disease. Arch Ophthalmol 1984;102:598-604.

37 Heffernan JT, Futterman S, Kalina R. Dexamethasone inhibition of experimental endothelial cell proliferation in retinal venules. Invest Ophthalmol Vis Sci 1978;17:565.

38 Rozen VB, Chernin LS. Effect of different doses of glucocorticoids on growth of monolayer cultures of connective tissue. Fed Proc 1974;24:861.

39 Weijtens O, Van der Sluijs FA, Schoemaker RC, et al. Peribulbar corticosteroid injection: vitreal and serum concentrations of the dexamethasone disodium phosphate injection. Am f Ophthalmol 1997;123:35863.

40 Weijtens O, Schoemaker RC, Cohen AF, et al. Dexamethasone concentration in vitreous and serum after oral administration. Am $\mathcal{F}$ Ophthalmol tion in vitreous

41 Cheng CK, Berger A, Pearson PA, et al. Intravitreal sustained-release dexamethasone device in the treatment of experimental uveitis. Invest Ophthalmol Vis Sci 1995;36:442-53.

42 Taba KE, Rochas JC, Spee C, et al. Intravitreal sustained release fluocinolone implant inhibits experimental choroidal neovascularization. Invest Ophthalmol Vis Sci 1999;40:S172.

43 Yang CS, Khawly JA, Hainsworth DP, et al. An intravitreal sustained-release triamcinolone and 5-fluorouracil codrug in the treatment of experimental proliferative vitreoretinopathy. Arch Ophthalmol 1998;116:69-77.

44 Gallemore RP, Yang $\mathrm{CH}$, Guo H, et al. Inhibition of proliferative vitreoretinopathy (PVR) using a fluocinolone/5-fluorouracil codrug sustained release pellet. Invest Ophthalmol Vis Sci 1997;38:S211.

45 Velez G, Robinson MR, Durbin T, et al. Thalidomide sustained release devices for choroidal neovascularization: an in-vitro analysis. Invest Ophthalmol Vis Sci 1999;40:S84.

46 Ip MS, Reichel E, Vieira W, et al. Neuroprotective effect of a sustained intravitreal calcium channel blocker in elevated IOP in the rabbit. Invest Ophthalmol Vis Sci 1998;39:S275.

47 Netland PA, Chatuverdi N, Dreyer EB. Calcium channel blockers in the management of low-tension and open-angle glaucoma. Am f Ophthalmol 1993;115:608-13.

48 Tomita K, Araie M, Tamaki Y, et al. Effects of nilvadipine, a calcium antagonist, on rabbit ocular circulation and optic nerve head circulation in NTG subjects. Invest Ophthalmol Vis Sci 1999;40:1144-51.

49 Piltz JR, Bose S, Lanchoney D. The effect of nimodipine, a centrally active calcium channel antagonist, on visual function and macular blood flow in patients with normal-tension glaucoma and control subjects. F Glaucoma 1998;7:336-42.

50 Durbin TD, Robinson MR, Yuan P, et al. Sustained-release devices with trimetrexate for the treatment of intraocular lymphoma: an in-vitro study. Invest Ophthalmol Vis Sci 1999;40:S85.

51 Bleyer WA. The clinical pharmacology of methotrexate. Cancer 1978;41:3651 .

52 Physician's desk reference. 53nd ed. Montvale, NJ: Medical Economics Company, 1999:3172-4.

53 Cheng L, Hostetler KY, Gardner MF, et al. Intravitreal toxicology in rabbits of two preparations of 1-O-octadecyl-sn-glycerol-3-phosphonoformate, a sustained-delivery anti-CMV drug. Invest Ophthalmol Vis Sci 1999;40: 1487-95.

54 Anglade E, Csaky KG. Recombinant adenovirus-mediated gene transfer into the adult rat retina. Curr Eye Res 1998;17:316-21

55 Csaky KG, Nussenblatt RB. Gene therapy in the treatment of ocular inflammation. Springer Sem Immunopathol 1999 (in press). 